\title{
Airborne Experiment for Soil Moisture Retrieval using GNSS Reflectometry
}

\author{
Hamza Issa ${ }^{1,2}$, Georges Stienne ${ }^{1}$, Serge Reboul ${ }^{1}$, Maximilian \\ Semmling ${ }^{3}$, Mohamad Raad ${ }^{4}$, Ghaleb Faour ${ }^{2}$ and Jens Wickert ${ }^{3}$ \\ ${ }^{1}$ Laboratoire d'Informatique, Signal et Image de la Côte d'Opale (LISIC, EA 4491), \\ Université du Littoral Côte d'Opale (ULCO), F-62228 Calais, France. \\ ${ }^{2}$ National Council of Scientific Research (CNRS-L), Remote Sensing Research \\ Center, 22411 Mansouriyeh, Lebanon. \\ ${ }^{3}$ German Research Centre for Geosciences GFZ, Potsdam, Germany \\ ${ }^{4}$ Department of Engineering, Lebanese International University, Mouseitbeh - PO \\ Box: 146404 Mazraa, Beirut-Lebanon. \\ \{hamza.issa,georges.stienne,serge.reboul\}@univ-littoral.fr, \\ \{maxsem,wickert\}@gfz-potsdam.de,mohamad.raad@liu.edu.lb and \\ gfaour@cnrs.edu.lb.
}

Measurement of soil moisture content on a global scale have gained increased interest over the years, due to its essential role in agriculture and most importantly in predicting the occurrence of natural disasters. This paper is dedicated to a study on GNSS Reflectometry (GNSS-R) using a low-altitude airborne carrier for soil moisture estimation with $1 \mathrm{~ms}$ rate of carrier-to-noise ratio observations. The principle of GNSS-R is the exploitation of L-band navigation signals as sources of opportunity to characterize the earth surface, because the reflected signals are often affected by the nature of the reflective surface. To scan large regional surface areas and quickly reach the areas to monitor, a dynamic GNSS-R system is considered.

The GNSS-R setup used in this study consists of RHCP and LHCP antennas mounted on the nose of a gyrocopter and of a Syntony front-end GNSS receiver. In addition, the gyrocopter is equipped with a signal digitizer and mass storage devices for digitizing and storing the base-band GNSS direct and reflected signals along the flight. A drone sensors board is also attached to the gyrocopter, which records the gyrocopter's attitude and position at $1000 \mathrm{~Hz}$ rate along its trajectory. To cope with the rapid displacement of the satellites' footprints along the receiver trajectory, high rate $(1 \mathrm{~ms})$ of carrier-to-noise ratio observations are processed with the data collection of the base-band RHCP and LHCP signals.

In the context of the study, it is very important to localize the reflective surfaces (satellites' footprints) from which each processed signal has reflected, and thus detect which areas were scanned during the flight. The link between the reflected signals and the satellites' footprints is based on the GPS time, attitude and position provided by the drone board and the GPS time extracted from the digitized GNSS signals. We show that these parameters allow to determine, at ms rate, the satellites' footprints locations (i.e. the surface areas) from which each signal has reflected at a specific GPS Time. A Geographic Information System is developed based on this principle to map the measurements obtained from the GNSS-R airborne setup along a real receiver trajectory. The ultimate aim of this study is to link the obtained GNSS-R measurements with the 
scanned surfaces to provide a soil moisture mapping of the studied area. 